\title{
ZERO-DISPLACEMENT END TERMINATION OF UNDULATORS AND WIGGLERS
}

\author{
S.C. Gottschalk, D.C. Quimby, K.E Robinson* \\ STI Optronics, Inc., Bellevue, WA 98004 USA \\ *Presently with Lawrence Berkeley National Laboratory.
}

\begin{abstract}
One of the more challenging aspects of hybrid permanent magnet undulator design is end-field termination. Klaus Halbach provided indication of correct excitation patterns for tapered wigglers', but implementation on hybrid undulators has proven more difficult. Initial results on APS undulators achieved complete passive gap dependence of both total residual angle and trajectory through the device, but with an entrance displacement. ${ }^{2}$ We have further refined the end field termination system by employing both partial volume and partial strength magnets. This achieves an entrance field configuration that maximizes the number of full strength poles without entrance or exit displacement, independent of the magnetic gap. This is all achieved with passive compensation techniques. End pole tuning is then used to correct small $(0.2 \%)$ deviations in the measured versus calculated end field steering. We present the design approach and results of five devices that have used this configuration.
\end{abstract}

\section{INTRODUCTION}

There have been a number of end field termination methods proposed. STI has used partial strength end magnets since $1986,{ }^{3}$ as well as partial strength and reduced height poles for the ID's delivered to APS. $\mathrm{Halbach}^{4}$ proposed variable excitation ends for tapered wigglers, ALS extended this to include rotatable end magnets ${ }^{5}$ and $\mathrm{ESRF}^{6}$ has used adjustable positioning of the initial poles as well. One challenge has been to come up with a design which maximizes the number of spectrally useful poles without also displacing the trajectory off axis.

We first demonstrated zero displacements ends on the fixed gap TJNAF $2.2 \mathrm{~cm}$ period IR FEL undulator in 1997. Two dimensional analyses indicated that zero displacement designs would be gap independent. This was confirmed experimentally on $55 \mathrm{~mm}$ and $33 \mathrm{~mm}$ period APS Undulator A ID's. We made several improvements to the design for the U9 device.

Heating magnets to reduce their field strength hampered our initial attempts to model end fields. We found that some magnets would cause a $300 \mathrm{G}-\mathrm{cm}$ steering change when the dipole strength of the first magnet was reduced from $90 \%$ to $80 \%$, but other magnets would cause no steering change at all. The reason is that reducing the dipole strength by heating always makes the magnetization nonuniform. The open circuit demagnetization of a magnet has larger demagnetizing fields at the center of the magnet than the edges. When the magnet is heated to reduce it's dipole moment, the center portion suffers a larger irreversible magnetization loss than the edges. We tried heating magnets in a closed circuit configuration because then the entire magnet is at the same operating point. This failed because such high temperatures were needed that the plating came off the magnets before we were even able to reduce their strength $10 \%$. We have found that the best method is to simply reduce the magnet volume.

There was a trade-off made between maintaining a large number of spectrally useful poles at all gaps and trajectory displacement. Our early APS end field designs maximized the number of useful poles at minimum gap, but at the expense of a large trajectory displacement. At the request of APS we changed the design to favor low displacement, but three poles were made partial strength. It was not possible to achieve both a large number of useful poles and small trajectory displacements if only the strength of the $1^{\text {st }}$ magnet and height of the $1^{\text {st }}$ two poles were varied. Another adjustment was needed: varying more magnet strengths was found to be sufficient.

\section{DESIGN METHOD}

There were two explicit goals in the end field design: 1) zero trajectory displacement and 2) maximize the number of spectrally useful poles over the spectral tuner's range. We achieved both goals by adjusting the volume of the $1^{\text {st }}$ magnet and the strength of the $2^{\text {nd }}$ and $3^{\text {rd }}$ magnets.

Earlier end field analyses ${ }^{2}$ showed that 2D FEA could be used for trajectory displacement calculations even in the presence of 3D effects such as non-zero entrance angles. The design approach we followed was to use 2D FEA for initial determinations of the magnet strengths. Refinements like changing the volume of the magnet were then added as needed. Finally we confirmed the design with a 10 pole $3 \mathrm{D}$ FEA.

The following description is for an example designused for the U9/SRRC undulator. ${ }^{8}$ We show in Figure 1 a correlation of trajectory displacement at $\mathrm{K}=10$ and $\mathrm{K}=1$ for a set of 7 different combinations of end magnet volumes and strengths. There is a clear linear correlation between small and large gap trajectory displacements. A zero displacement end will not change by more than 500 G- $\mathrm{cm}^{2}$ over a very wide gap tuning range. This is not true for non-displacing ends: for example a $20,000 \mathrm{G}-\mathrm{cm}^{2}$ end at $\mathrm{K}=10$ will become an $18,100 \mathrm{G}-\mathrm{cm}^{2}$ end at $\mathrm{K}=1$. Clearly it will be sufficient to design the end at only one gap. 


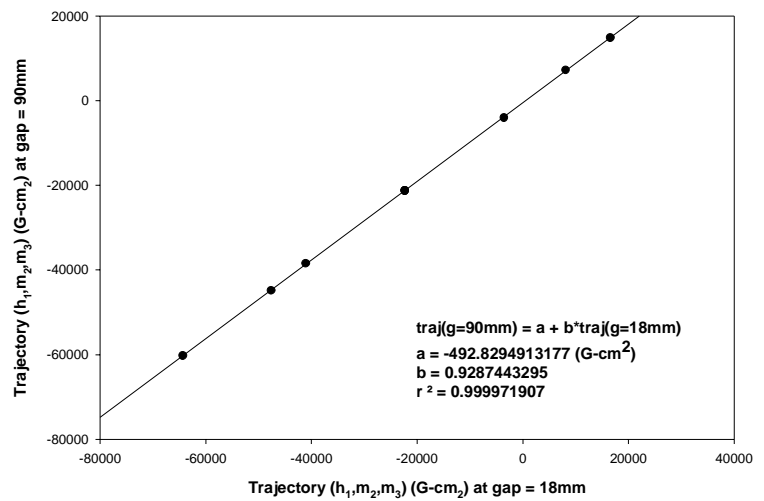

Figure 1. Linear regression of trajectory displacement at $\mathrm{g}=18 \mathrm{~mm}$ against $\mathrm{g}=90 \mathrm{~mm}$.

Both the trajectory displacements as well as the peak field values are linear in the volume of the $1^{\text {st }}$ magnet and the strengths of the $2^{\text {nd }}$ magnet. We chose to keep the clamped surface of the $1^{\text {st }}$ magnet at the same location as all the other magnets but to vary its vertical height. After the $5^{\text {th }}$ pole, the remaining poles were insensitive to details of the ends. It was found that there were an infinite number of zero displacement ends. The only condition was that the height of the $1^{\text {st }}$ magnet varies linearly with the strength difference between the $3^{\text {rd }}$ and $2^{\text {nd }}$ magnets, see Figure 2. Imposing a requirement on the $3^{\text {rd }}$ peak gave a unique solution. The optimization result is shown in Figure 3. The combination in which the $3^{\text {rd }}$ magnet is $95 \%$ of full strength can achieve zero displacement, but the $3^{\text {rd }}$ and $4^{\text {th }}$ peaks are imbalanced. Making the $3^{\text {rd }}$ magnet full strength gave peak values of $100 \%, 99.91 \%$ and $99.86 \%$ for poles 3,4 and 5 respectively. The dipole strength dispersion needed to achieve this was $9.5 \%$. We decided to reduce the strength of the $2^{\text {nd }}$ magnet to $90.5 \%$ by heating since such a small strength reduction should not produce any large magnet inhomogeneities.

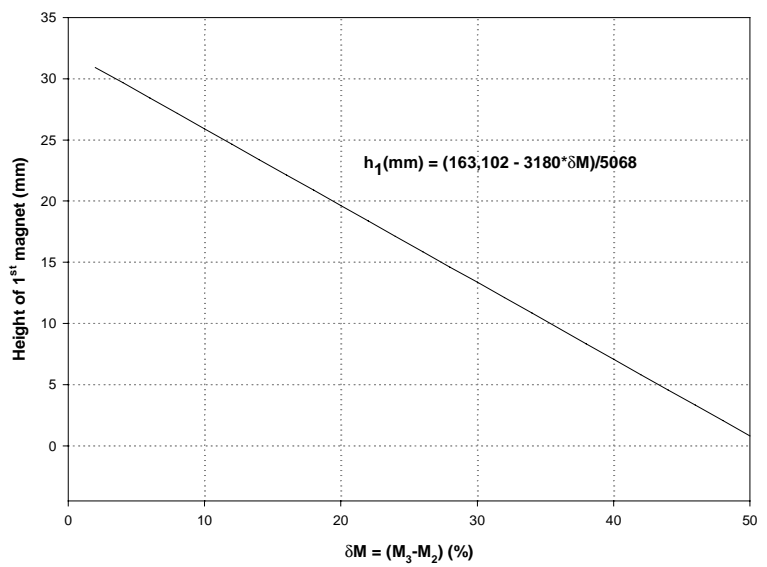

Figure 2. Zero trajectory displacement; entrance conditions on $\mathrm{h}$ and $\delta \mathrm{m}$, gap $=18 \mathrm{~mm}, \mathrm{~K}=10$.

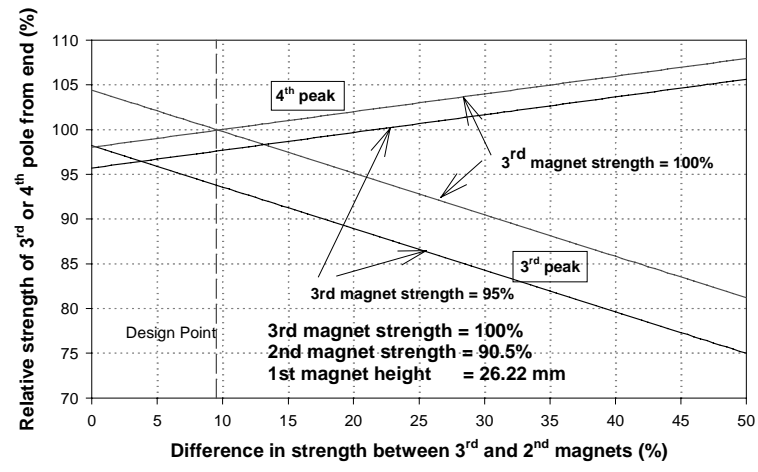

Figure 3. Relative peak field srengths of $3^{\text {rd }}$ and $4^{\text {th }}$ poles from end zero trajectory displacement, gap $=18 \mathrm{~mm}$,

$$
\mathrm{K}=10 \text {. }
$$

\section{RESULTS}

The calculated end field and trajectory using 2D FEA is shown in Figure 4. The final part of the analysis was to include side magnets. A side magnet that is attached to a pole will steer the e-beam very strongly, especially on the ends. The 3D FEA prediction was that a side magnet at the $2^{\text {nd }}$ pole would cause xxx G-cm of steering, but reducing the side magnet strength to $50 \%$ would eliminate the steering. We placed side magnets on one side of the upper and lower poles to give a 50\% steering effect without causing any changes in normal or skew multipoles. Experimental confirmation of the end design for $\mathrm{K}=10$ and $\mathrm{K}=1$ are shown in Figure 5.
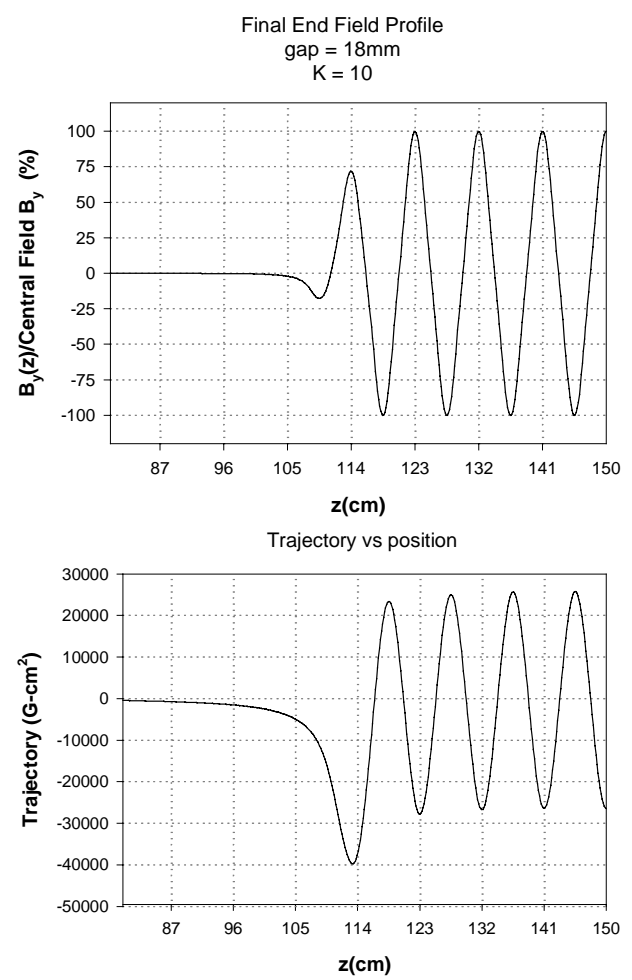

Figure 4. Field profiles and trajectory for final end field configuration. 


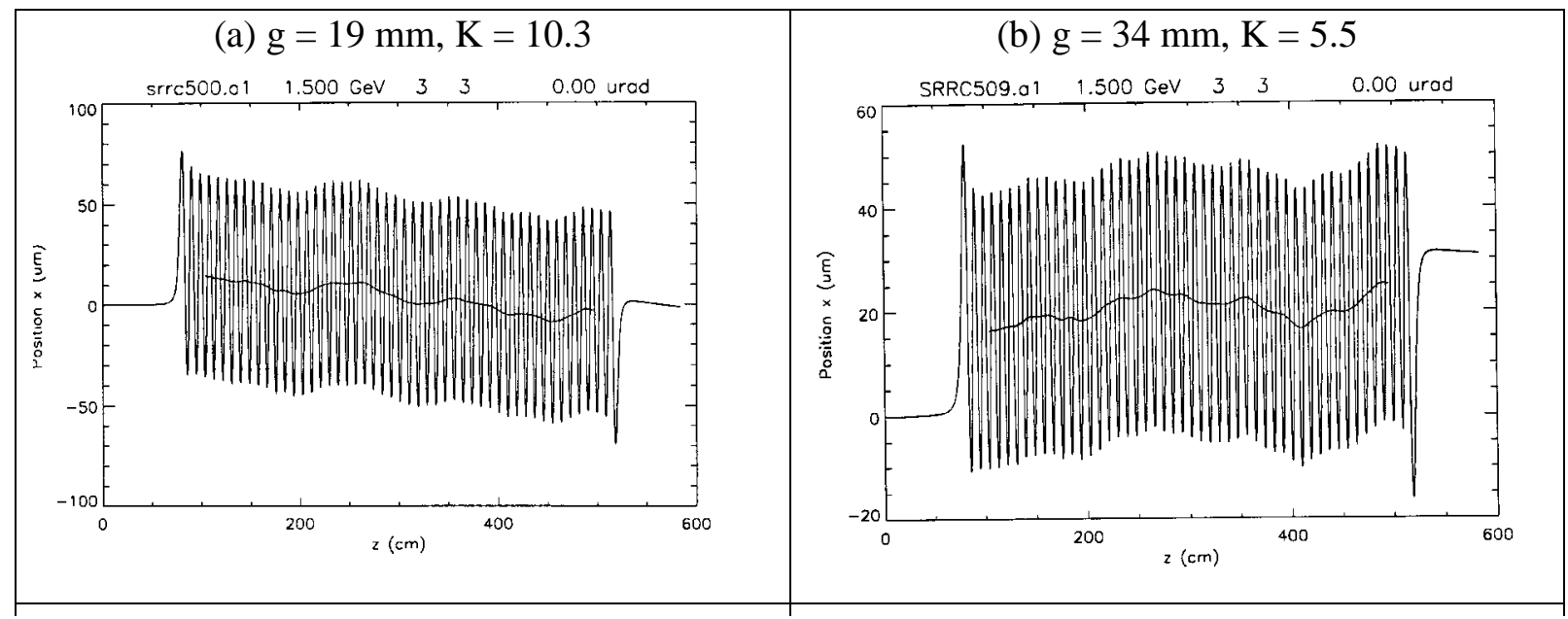

Figure 5. Measured electron trajectories and calculated spectral intensity at gaps $=19 \mathrm{~mm}$ and $34 \mathrm{~mm}$.

\section{CONCLUSION}

We have demonstrated that gap independent zero displacement end fields can be achieved by varying the strength of the initial magnets. The design was chosen to produce a large number of spectrally useful poles. At minimum gap $(\mathrm{K}=10)$ only two poles at each end were not spectrally useful while at a large gap $(\mathrm{K}=1)$ only three poles were not useful. We are investigating the feasibility of further improvements if the shapes and/or locations of the last few poles are varied as well. The design goal would be to maintain resonance over as many poles as possible.

\section{ACKNOWLEDGEMENTS}

The authors would like to acknowledge the contributions of the employees of STI to the construction and measurements of the U9/SRRC device. The advise of T.-C. Fan, C.-H. Chang, C.-S. Hwang and J. Chen of SRRC are sincerely appreciated.

\section{REFERENCES}

[1] K. Halbach, private communication. Comprehensive summary given by R. Schleuter, $2^{\text {nd }}$ ICFA School of Synchrotron Light Sources, October, 1993.

[2] S.C. Gottschalk, K.E. Robinson, I. Vasserman, R. Dejus, and E.R. Moog, "End Field Design and Tuning Methods for Insertion Devices," Rev. Sci. Instr. 67 (1996), Paper C)9 (CDROM issue).

[3] K.E. Robinson, D.C. Quimby, and J.M. Slater, IEEE J. Quantum Electronics, QE-23 (1987) 1497.

[4] "W16.0 Undulator Conceptual Design Report", Lawrence Berkeley Laboratory Publication 5256 (November, 1989).

[5] U5.0 Undulator Conceptual Design Report, Lawrence Berkeley Laboratory Publication 5256 (November, 1989).

[6] J. Chavanne, P. Elleaume,, and P. Van Vaerenergh, "Status of the ESRF Insertion Devices," Reev. Sci. Insr. 67 (1996), Paper C04 (CD-ROM issue).

[7] S.V. Benson, "High Average Power Free Electron Lasers," presented at this conference.

[8] D.C. Quimby, S.C. Gottschalk, D.R. Jander, T.E. DeHart, K.E. Robinson, A.S. Valla, and J.F. Zumdieck, " The 90-mm Period Undulator for SRRC, presented at this conference. 